Instituto Internacional de Investigación y Desarrollo Tecnológico Educativo INDTEC, C.A.

DOI: https://doi.org/10.29394/Scientific.issn.2542-2987.2021.6.20.18.327-339

OAI-PMH: http://www.indteca.com/ojs/index.php/Revista Scientific/oai

Ensayo Original / Original Essay

\title{
Equidad de género en la educación superior en Ecuador: Aproximación a su conceptualización
}

\author{
Autores: Jomar Elizabeth Guzmán Seraquive \\ Magisterio Fiscal \\ jomaliz-1991@hotmail.com \\ Guayas, Ecuador \\ https://orcid.org/0000-0002-8387-8921
}

Carlos Vicente Jones Ortiz Universidad Nacional de Educación, UNAE carlosv jones93@hotmail.com Azogues, Ecuador https://orcid.org/0000-0002-3132-7765

\section{Resumen}

La equidad de género desde la perspectiva educativa, es una de las condiciones fundamentales para garantizar la integración de diferentes sectores poblacionales que de otra manera se verían vulnerados a recibir una educación eficiente y de calidad. El ensayo se basa en la percepción y criterio de diversos autores como Baute, Pérez y Luque (2017); Bell (2017); Mayorga (2018); Buquet (2016); Pessina (2017); Fuentes (2016), buscando la mitigación y reducción de las diferencias en base a la transversalidad del género desde el proceso de evaluar las consecuencias, sobre todo en el ámbito educativo. Si bien en los últimos años se ha trabajado y potencializado un sinnúmero de aspectos relacionados a la igualdad, son varios los esfuerzos de los sistemas educativos en este sentido, aún se evidencian necesidades de acciones para lograr una total equidad. El objetivo se fundamenta en analizar diferentes aspectos, criterios y conceptos que abordan la igualdad de género en la educación superior en el Ecuador, contrastando el progreso alcanzado, las falencias que aún se presentan y acciones que se puedan llegar a tomar.

Palabras clave: equidad de género; educación superior; sociedad. Código de clasificación internacional: 6310.99 - Equidad de género.

\section{Cómo citar este ensayo:}

Guzmán, J., \& Jones, C. (2021). Equidad de género en la educación superior en Ecuador: Aproximación a su conceptualización. Revista Scientific, 6(20), 327-339, e-ISSN: 2542-2987. Recuperado de: https://doi.org/10.29394/Scientific.issn.2542-2987.2021.6.20.18.327-339

Fecha de Recepción: 15-01-2021
Fecha de Aceptación: 19-03-2021
Fecha de Publicación: 05-05-2021 
Instituto Internacional de Investigación y Desarrollo Tecnológico Educativo INDTEC, C.A.

DOI: https://doi.org/10.29394/Scientific.issn.2542-2987.2021.6.20.18.327-339

OAI-PMH: http://www.indteca.com/ojs/index.php/Revista Scientific/oai

Ensayo Original / Original Essay

\title{
Gender equity in higher education in Ecuador: Approach to its conceptualization
}

\begin{abstract}
Gender equity from the educational perspective is one of the fundamental conditions to guarantee the integration of different population sectors that would otherwise be compromised to receive an efficient and quality education. The essay is based on the perception and criteria of various authors such as Baute, Pérez and Luque (2017); Bell (2017); Mayorga (2018); Buquet (2016); Pessina (2017); Fuentes (2016), seeking the mitigation and reduction of differences based on gender mainstreaming from the process of evaluating the consequences, especially in the educational field. Although in recent years a number of aspects related to equality have been worked on and promoted, there are several efforts made by education systems in this regard, there are still needs for actions to achieve total equity. The objective is based on analyzing different aspects, criteria and concepts that address gender equality in higher education in Ecuador, contrasting the progress achieved, the shortcomings that still exist and actions that may be taken.
\end{abstract}

Keywords: gender equality; higher education; society. International classification code: 6310.99 - Gender equality.

How to cite this essay:

Guzmán, J., \& Jones, C. (2021). Gender equity in higher education in Ecuador: Approach to its conceptualization. Revista Scientific, 6(20), 327-339, e-ISSN: 2542-2987. Recovered from: https://doi.org/10.29394/Scientific.issn.2542-2987.2021.6.20.18.327-339

Date Received: 15-01-2021
Date Acceptance: 19-03-2021
Date Publication: 05-05-2021 


\section{Ensayo Original / Original Essay}

\section{Introducción}

Equidad de género es un concepto afinado y forjado a consecuencia de múltiples prácticas y organizaciones de mujeres que durante los últimos años se han encargado de elaborar agendas de justicia genérica. En este sentido el progreso del concepto de igualdad de género es testigo de una dialéctica en la interrelación de la progresiva influencia del movimiento social globalizado decidido a la modificación de las relaciones desiguales entre sexos y la elaboración teórica del descubrimiento y visibilización de la opresión femenina.

En este sentido, Mayorga (2018): menciona que se puede definir a la equidad de género como un conjunto de normas, valores personales que determinan la identidad de cada individuo aportando a cada oportunidad que estos tienen. El término género asociado a la igualdad tanto para hombres como para mujeres, es una noción sobre lo que en hoy en día se debate de manera abierta en diferentes medios de nuestra sociedad. Más allá del enfoque mediático y la escasa claridad conceptual del mismo, es importante realizar una revisión de los diferentes puntos de vista teóricos que lo definen.

Para Baute, Pérez y Luque (2017a): la posición de la equidad de género trata de evidenciar las posibilidades que tienen las mujeres frente a los hombres, en el ámbito educativo, trata de reflejar si existe algún tipo de desventaja con respecto uno del otro.

Se puede inferir que las mujeres y los hombres poseen características y oportunidades diferentes, este punto debe ser analizado para en el caso de existir desigualdades referentes al acceso a la educación se rectifique. Ya sea aplicando políticas o estrategias que partea desde el gobierno central y pasen a las universidades.

El concepto de género cuestiona como la sociedad tal y como se encuentra conformada, desde un punto de vista masculino se ha minimizado a las mujeres y los aportes de estas en muchas esferas de la vida social, relegándolas a escalas de lo privado o el hogar. Los estudios sobre equidad 
de género surgen en la educación superior, sobre todo como una protesta a las posiciones teóricas tradicionales que ignoran o distorsionan la experiencia de la mujer desconociendo su contribución a la generación de conocimiento.

El objetivo del presente ensayo es diferentes aspectos, criterios y conceptos que abordan la igualdad de género en la educación superior en el Ecuador, contrastando el progreso alcanzado, las falencias que aún se presentan y acciones que se puedan llegar a tomar.

\section{Desarrollo}

\subsection{El tema de la mujer al centro del enfoque de equidad de género}

El análisis y la ejemplificación de algunas de las principales posibilidades que la inclusión educativa ha sido motivo de arduo debate por parte de los organismos interesados. La inclusión y la igualdad de género ha sido el principal desafío de las entidades escolares.

Las políticas referentes a la educación, tienen inmersos puntos de vista de igualdad, ya sea en las líneas pedagógicas o en el trato del individuo, donde se persigue evitar la discriminación de cualquier tipo. Paralelamente, Bell (2017): indica que "la inclusión educativa representa uno de los principales desafíos para las instituciones de Educación Superior en los momentos actuales y demanda la integración e intensificación de las acciones [...]" (pág. 200).

En este aspecto, Fuentes (2016): dice que en el caso de las mujeres se evidencio un acceso tardío a la educación respecto a los hombres, esto como referencia al siglo XX. Se piensa que la educación es el único medio para la superación tanto personal como profesional, a partir de este pensamiento surgió la incursión de las mujeres de forma significativa en la educación universitaria donde para aquella época era predominante el género masculino.

Las mujeres lograron ingresar de forma masiva a la educación superior en carreras del área médica, educativas, las cuales eran acordes al género, 


\section{Ensayo Original / Original Essay}

mientras que en el área de ingeniera eran reconocidas como netamente para hombres.

Según Buquet (2016): pese a que en los últimos años se ha visto un avance en el número de mujeres universitarias, la tasa de participación sigue siendo menor en relación con el conjunto de etapas del sistema educativo. Si bien las mujeres han ganado en formación, en países europeos y sobre todo latino americano, no se ha incrementado de manera significativa el número de mujeres en las instituciones de enseñanza debido a las condiciones de desigualdad, el género sigue siendo un factor determinante en el proceso de profesionalización.

Parafraseando a Winfield, Jiménez y Topete (2017): debido a la presión y otros medios de acción, se abrió una puerta a la vida académica de las mujeres, no solo como parte del alumnado sino también como docentes e investigadoras de prestigio y con proyección hacia los puestos de gestión. Pero a pesar de ello queda mucho por recorrer en cuanto al acceso a la presencia de la mujer en los gobiernos universitarios.

Quizá el principal aspecto de esta evolución es precisamente la eliminación de entidades de educación segregadas, que transmitían un modelo de formación diferenciado, debido a la separación de sexos, bien masculino o femenino, una modalidad establecida en funciones retrógradas de un principio que desaconsejaba la convivencia de mujeres y hombres en espacios que no fuesen domésticos o familiares.

Así mismo se expone la necesidad de eliminar los modelos socioculturales que separan a hombres y mujeres en el sector educativo, social o cultural, ya que esa discriminación que se genera en los niños, perdura en el tiempo afectando su conducta profesional en el futuro.

Las mujeres ecuatorianas representan un gran avance en el acceso a la educación en los últimos 20 años, según expone la ONU Mujeres Ecuador (2015): en el informe Mujeres ecuatorianas, dos décadas de cambio, la 
Instituto Internacional de Investigación y Desarrollo Tecnológico Educativo INDTEC, C.A.

DOI: https://doi.org/10.29394/Scientific.issn.2542-2987.2021.6.20.18.327-339

OAI-PMH: http://www.indteca.com/ojs/index.php/Revista Scientific/oai

Ensayo Original / Original Essay

representación de la mujer pasa a ser mayoría en Economía y en Ciencias Políticas y aumenta en área de Arquitectura y legislación; pero se minimiza el ingreso en la informática y Ciencias Exactas donde las mujeres siempre han sido minorías

Señalando a Mantilla-Falcón, Galarza-Galarza y Zamora-Sánchez (2017): la educación superior ha experimentado positivos cambios, en el Ecuador, así como también en países latinoamericanos, pero a pesar de ello el sexismo no se ha erradicado del todo. Por consiguiente, esto genera la desigualdad marcada entre ambos sexos, predominando la idea que existen carreras para hombres y mujeres.

Según los autores se ha marcado una constante mejoría en la situación de la mujer en las Universidades, sin embargo, concuerdan también que el sexismo y otros factores como la diversidad étnica aún enmarcan y dificultan su vida estudiantil.

En la Constitución de la República del Ecuador (CRE, 2008): se expresa que "nadie podrá ser discriminado por razones de etnia, lugar de nacimiento, edad, sexo, identidad de género, identidad cultural, estado civil, idioma, religión, ideología, filiación política, pasado judicial, condición socioeconómica, condición migratoria, orientación sexual, estado de salud, portar VIH, discapacidad [...]" (pág. 12). Es importante considerar la educación como un medio para logar la igualdad de género, como un factor de superación de la marginación social, económica y profesional que puede sufrir una mujer.

En este mismo orden de ideas, Menéndez, Venegas, Bermeo y Peñafiel (2017): mencionan que el Ecuador las instituciones de educación superior son el factor primordial para lograr alcanzar una equidad de género, siendo así que la presencia femenina en el ámbito universitario, tiene una tendencia ascendente en los últimos años, esto acompañado del fenómeno general de crecimiento de matriculación. Lo que conlleva a pensar que se ha pasado de una presencia minoritaria a una más equivalente al género masculino. 
Conscientemente la persistencia en la desigualdad estructural en la sociedad moderna ha contribuido sin dudas a hacer de la Educación Superior, quien debiera ser el lugar por excelencia de investigación científica, un campo elitista que profundiza también en las desigualdades de género. En esa misma línea, Pessina (2017): refiere que además de las brechas sociales, la discriminación laboral, o la violencia en la vida diaria, en el área de la ciencia es donde las mujeres parecen enfrentarse a nuevas formas de discriminación.

De acuerdo con Baute, Pérez y Luque (2017b): la Universidad contemporánea en el Ecuador tiene entre sus objetivos fundamentales el cambio de la sociedad a partir de políticas como la aplicación del Plan Nacional del Buen Vivir que contiene las bases de la interculturalidad. La inclusión asume un rol importante en tema de igualdad, ya que se considera que un pueblo educado tiende a minimizar las barreras existentes.

Por lo mencionado anteriormente se puede deducir que el tema de la educación es un aspecto primordial en la incursión de las mujeres al mundo laboral. La participación de mujeres y hombres en los procesos de producción y su incursión en las actividades científicas y tecnológicas están claramente condicionadas, entre otras cosas, por su desarrollo e incorporación oportuna a la formación universitaria.

Como exponen Martínez, Rodríguez y García (2018): "el Estado ecuatoriano asume, como una de sus principales responsabilidades y metas, la transformación del sistema educativo en todos los niveles, hasta alcanzar los estándares de eficiencia y calidad que hagan de la educación un verdadero instrumento de equidad, desarrollo sostenible y calidad [...]" (pág. 161).

A continuación, en la tabla 1 se presenta la tasa neta de escolarización superior de hombres y mujeres tomando en consideración el sector urbano y rural los datos fueron tomados del último censo realizado en el Ecuador: 
Tabla 1. Tasa Neta de escolarización superior según el último censo nacional en Ecuador (Censos de población y vivienda).

\begin{tabular}{|l|c|c|}
\hline \multicolumn{1}{|c|}{ Área } & Mujeres & Hombres \\
\hline Urbana & $68,2 \%$ & $62,7 \%$ \\
\hline Rural & $46,2 \%$ & $38,5 \%$ \\
\hline Nacional & $62,2 \%$ & $55,7 \%$ \\
\hline
\end{tabular}

Fuente: Ferreira, García, Macías, Pérez y Tomsich (2011).

En estos datos, el acceso a la educación en instituciones de educación superior para el sector urbano presenta un porcentaje del $68,2 \%$ para las mujeres, mientras que los hombres $62,7 \%$, en la zona rural con un $46,2 \%$ para el género femenino y para el género masculino un $38,5 \%$, siguiendo esta tendencia a nivel nacional se tiene un $62,2 \%$ para las mujeres frente a un $55,7 \%$ para los hombres, esto lleva a denotar que ha existido un incremento de la escolarización de mujeres frente a los hombres, si bien las mujeres han ganado en formación, en el país no se ha incrementado de manera significativa su participación en las instituciones de enseñanza debido a las condiciones de desigualdad, el género sigue siendo un factor definitivo en el proceso de profesionalización.

Gráfico 1. Tasa neta de escolarización superior.

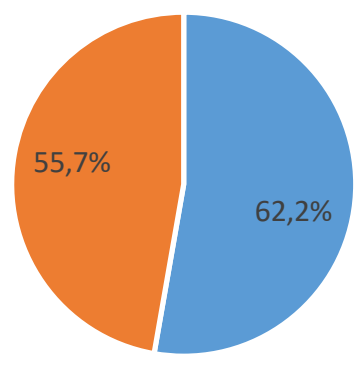

- Mujeres - Hombres

Fuente: Ferreira, García, Macías, Pérez y Tomsich (2011). 
En el gráfico 1, se denota el acceso a la educación superior a nivel nacional en la relación a las mujeres refleja un porcentaje del $62,2 \%$ frente a un $55,7 \%$ de los hombres, refleja un importante avance en lo que concierne a la equidad de género a pesar de que los datos son tomados del último censo en el Ecuador.

Adicionalmente, Ordorika (2015): manifiesta que "algunas instituciones han planteado la equidad de género como parte de sus principios fundamentales, establecidos en sus estatutos y reglamentos; además han formulado políticas y lineamientos para alcanzar la equidad [...]" (pág. 11). Lo que conlleva minimizar el grado de desigualdad gracias a los reglamentos que se implementan. Partiendo de lo propuesto, se puede pensar que, en diferentes aspectos, es cuestión de tiempo, para que los planes de igualdad estén definidos en leyes y artículos en las constituciones de cada país.

\section{Conclusiones}

La igualdad de oportunidades para ambos sexos desde el ámbito socioeducativo considera el papel de competencias y habilidades de inclusión laboral y social. Pese a los avances que se han dado en cuanto a la desigualdad, las oportunidades que se generan para las mujeres en busca de la equidad de género siguen siendo limitadas.

Se ha vuelto un desafío colectivo asociado al mejoramiento de la calidad de la educación y la superación continua de las desigualdades que pudieran afectarla, se han resuelto diferentes consensos en varios frentes como medida de desarrollo.

\section{Referencias}

Baute, M., Pérez, V., \& Luque, M. (2017a,b). Estrategia de equidad de género en el entorno Universitario. Universidad y Sociedad, 9(4), 5057, e-ISSN: 2218-3620. Recuperado de: 
https://rus.ucf.edu.cu/index.php/rus/article/view/664

Bell, R. (2017). EI Desarrollo de los Procesos Sustantivos de la Educación Superior Ecuatoriana ante el Reto de la Inclusión Educativa. Revista Latinoamericana de Educación Inclusiva, 11(1), 199-212, eISSN: 0718-7378. Recuperado de:

https://scielo.conicyt.cl/pdf/rlei/v11n1/art12.pdf

Buquet, A. (2016). El orden de género en la educación superior: una aproximación interdisciplinaria. Nómadas (Col), (44), 27-43, e-ISSN: 0121-7550. Recuperado de:

https://www.redalyc.org/articulo.oa?id=105146818003

CRE (2008). Constitución de la República del Ecuador. Registro Oficial 449 de 20-oct-2008. Ecuador: Asamblea Constituyente del Ecuador.

Ferreira, C., García, K., Macías, L., Pérez, A., \& Tomsich, C. (2011). Mujeres y Hombres del Ecuador en Cifras III. Quito, Ecuador: Instituto Nacional de Estadística y Censos; ONU Mujeres.

Fuentes, L. (2016). ¿Por qué se requieren políticas de equidad de género en la educación superior?. Nómadas, 44, 65-83, e-ISSN: 0121-7550.

Recuperado de: http://www.scielo.org.co/pdf/noma/n44/n44a05.pdf Mantilla-Falcón, L., Galarza-Galarza, J., \& Zamora-Sánchez, R. (2017). La Inserción de la Mujer en la Educación Superior Ecuatoriana: Caso Universidad Técnica de Ambato. Revista Latinoamericana de Estudios Educativos, 13(2), 12-29, e-ISSN: 1900-9895. Recuperado de: https://www.redalyc.org/articulo.oa?id=134154501002

Martínez, O., Rodríguez, M., \& García, W. (2018). Educación Continua de la UNAE: Un modelo que aporta a la Transformación Educativa del Ecuador: Estudios sobre Educación. Revista Scientific, 3(8), 159180, e-ISSN: 2542-2987. Recuperado de:

https://doi.org/10.29394/Scientific.issn.2542-2987.2018.3.8.8.159-180 Mayorga, K. (2018). Igualdad de Género en la Educación Superior en el 


\section{Ensayo Original / Original Essay}

Siglo XXI. Palermo Business Review, (18), 137-144, e-ISSN: 03285715. Recuperado de:

https://www.palermo.edu/economicas/cbrs/pdf/pbr18/PBR 18 07.pdf Menéndez, J., Venegas, L., Bermeo, F., \& Peñafiel, F. (2017). Perspectivas de género en la educación superior: políticas y lineamientos a partir de un enfoque académico. ReHuSo: Revista de Ciencias Humanísticas y Sociales, 2(4), 11-27, e-ISSN: 2550-6587. Recuperado de: https://revistas.utm.edu.ec/index.php/Rehuso/article/view/1236

ONU Mujeres Ecuador (2015). Mujeres Ecuatorianas dos Décadas de Cambios 1995 - 2015. 1ra. Edición. Quito, Ecuador: Grupo Impresor.

Ordorika, I. (2015). Equidad de género en la Educación Superior. Revista de la Educación Superior, 44(174), 7-17, e-ISSN: 2395-9037. Recuperado de:

http://www.scielo.org.mx/pdf/resu/v44n174/v44n174a1.pdf

Pessina, M. (2017). ¿La ciencia, cuestión de hombres? Mujeres entre la discriminación, los estereotipos y el sesgo de género. Primera Edición, ISBN: 978-9978-55-168-4. Quito, Ecuador: Ediciones Ciespal. Winfield, A., Jiménez, Y., \& Topete, C. (2017). Representaciones mentales y sociales en la equidad de género. Revista de estudios de género. La Ventana, (45), 186-210, e-ISSN: 2448-7724. Recuperado de: http://www.scielo.org.mx/pdf/laven/v5n45/1405-9436-laven-5-4500186.pdf 
Instituto Internacional de Investigación y Desarrollo Tecnológico Educativo INDTEC, C.A.

DOI: https://doi.org/10.29394/Scientific.issn.2542-2987.2021.6.20.18.327-339

OAI-PMH: http://www.indteca.com/ojs/index.php/Revista Scientific/oai

Ensayo Original / Original Essay

Jomar Elizabeth Guzmán Seraquive

e-mail: jomaliz-1991@hotmail.com

Nacida en Bucay provincia del Guayas, Ecuador, el 24 de

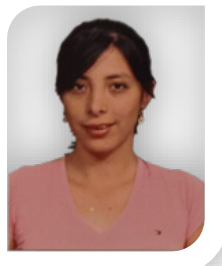
mayo del año 1991. Ingeniera en computación e informática por la Universidad Agraria del Ecuador (UAE); Tecnóloga en computación e informática por la Universidad Agraria del Ecuador; me desempeño como docente Titular del magisterio Fiscal; directora del área de matemáticas en la Unidad Educativa General Antonio Elizalde; conocedora de didáctica de la matemáticas y educación mediante las Tics. 


\section{Carlos Vicente Jones Ortiz} e-mail: carlosv jones93@hotmail.com

Nacido en Milagro, Ecuador, el 7 de mayo del año 1993.

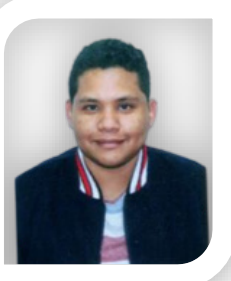
Máster en dirección e ingeniería de Sitios Web por la Universidad Internacional de La Rioja (UNIR), España; Ingeniero en Computación e Informática por la Universidad Agraria del Ecuador (UAE); me desempeño como analista de software en la Universidad Nacional de Educación (UNAE); investigador en temas educativos.

\footnotetext{
El contenido de este manuscrito se difunde bajo una Licencia de Creative Commons Reconocimiento-
} NoComercial-Compartirlgual 4.0 Internacional 